\title{
Let's Talk Learning Analytics: A Framework for Implementation in Relation to Student Retention
}

\author{
Deborah West and David Heath \\ Charles Darwin University \\ Henk Huijser \\ Xi'an Jiaotong-Liverpool University \& Batchelor Institute of Indigenous Tertiary Education
}

\begin{abstract}
This paper presents a dialogical tool for the advancement of learning analytics implementation for student retention in Higher Education institutions. The framework was developed as an outcome of a project commissioned and funded by the Australian Government's Office for Learning and Teaching. The project took a mixed-method approach including a survey at the institutional level $(n=24)$, a survey of individual teaching staff and other academics with an interest in student retention ( $\mathrm{n}=353)$, and a series of interviews $(n=23)$. Following the collection and analysis of these data an initial version of the framework was developed and presented at a National Forum attended by 148 colleagues from 43 different institutions. Participants at the forum were invited to provide commentary on the usefulness and composition of the framework which was subsequently updated to reflect this feedback. Ultimately, it is envisaged that such a framework might offer institutions an accessible and concise tool to structure and systematize discussion about how learning analytics might be implemented for student retention in their own context.
\end{abstract}

\section{Introduction}

This paper reports on findings from an Australian Government Office for Learning and Teaching commissioned project entitled Learning Analytics: Assisting Universities with Student Retention. One of the primary outputs of this project was a framework based on data collected from institutions and academics, using a mixed method approach featuring two different surveys and a series of interviews, conducted between July, 2014 and February, 2015. Following the surveys and interviews a draft 
framework of factors relevant to the institutional implementation of learning analytics for student retention purposes was developed. The framework was presented to peers and their feedback elicited at a National Forum held in April 2015, based on which the draft framework was refined to arrive at the version presented here.

The primary purpose of this paper is to present a framework of factors relevant to the institutional implementation of learning analytics, situate it with other thinking in this area, and discuss how the framework might be used in practice. Such a framework may prove a useful tool for institutions engaged in decision making and planning around the use of learning analytics for student retention purposes, particularly because learning analytics initiatives often require people with a wide variety of expertise to work together (e.g. coding and programming, project management, learning and teaching, discipline knowledge, statistics, and potentially a range of others). A secondary purpose is to explain the process of the framework's development and explore the context in which this development occurred. Attention to this second element is critical as it draws attention to the underpinning thinking linked to previous work in the field, and data input highlighting the need for a dialogical tool due to the complexity of learning analytics.

Structurally, the paper begins with a brief literature review in which the two primary concepts associated with the framework are explored: student retention and learning analytics. The literature review also considers other frameworks and tools related to institutional implementation of learning analytics. To conclude the literature review, the research questions and thinking behind these are described followed by the research methods. The results section focuses initially on headline findings from the data, which leads into presentation of the framework. The paper concludes with some propositions about how the framework might be used, discussion of how it fits with other conceptualisations of institutional implementation of learning analytics, and a brief overview of future directions.

\section{Literature Review}

\section{Student Retention}

Retention, and more broadly student success, are critical but challenging issues within the higher education sector in Australia. Widening participation agendas, which include the desire to have an increased number of students and a broader diversity of students, are a high priority (Bradley, Noonan, Nugent \& Scales, 2008). Key cohorts for improving higher education participation and success are Aboriginal and Torres Strait Islander people (Universities Australia, 2013; Behrendt, Larkin, Griew \& Kelly, 2012), people from regional areas and people of low socio-economic status (Universities Australia, 2013).

Responding to this challenge, institutions have been working toward adapting their environments and learner support mechanisms. One aspect of this has been the use of technology enabled learning, which can provide increased flexibility and personalised learning opportunities. Despite the efforts made to date, attrition rates continue to be high, particularly for more diverse learner cohorts with a sector average of $17.31 \%$ (Koshy, 2014). Additionally, there are concerns around higher attrition rates for students studying online (Dekker, Pechenizkiy \& Vleeshouwers, 2009; Rivera \& Rice, 2002). There is some speculation around the reasons for this including differences in student demographics, difficulties with effective use of technology and social isolation (Frankola, 2002). 
The academic and non-academic factors that can influence retention are complex and varied (Nelson, Clarke, Stoodley \& Creagh, 2014). In a study of business students across six Australian universities ( $\mathrm{n}$ 7486), Willcoxson and colleagues (2011: 1) reported the following:

...data strongly indicates that factors related to attrition are generally university-specific and reflect both student characteristics and their responses to the specific institutional culture and environment. The only attrition triggers which span most universities and most years of study are 'lack of a clear reason for being at university' and 'the feeling of having insufficient ability to succeed at university’.

This suggests that attempts to tackle attrition need to be considered and contextualised to specific institutions. It is widely recognised that retention is one facet of student success and that many of the actions that we take to improve outcomes for those students who are 'at risk' can actually benefit all students throughout their learning journey.

\section{Learning Analytics}

Linked to technology enabled learning and broader digitalisation of interactions (e.g. libraries, student services engagements) are a range of opportunities to collect and analyse data with the intention of improving the student experience (Gaševic, Dawson, \& Siemens, 2015). As Buckingham Shum and Ferguson have observed (2012: 3) "the core proposition is that, as unprecedented amounts of digital data about learners' activities and interests become available, there is significant potential to make better use of this data to improve learning outcomes". While there has been much debate around definitions of learning analytics and attempts to disaggregate learning analytics from educational analytics, educational data mining and academic analytics (Ferguson, 2012; Gaševic et al., 2015; Long \& Siemens, 2011; van Barneveld, Arnold, \& Campbell, 2012), such debates are not seen as useful in the context of this paper. Learning analytics is an emerging field and most in the sector are more interested in how data can be used rather than the nuances of definitions (Sclater, 2014). For our purposes, learning analytics can be seen as the "measurement, collection, analysis and reporting of data about learners and their contexts, for purposes of understanding and optimizing learning and the environments in which it occurs" (Long \& Siemens, 2011). Sclater (2014) notes that such data can be seen to be on a continuum of use value to different stakeholders (which is more in line with how institutions perceive it) and can be used by these various stakeholders (including students, tutors, educational researchers, unit heads and management) for different purposes, depending on their particular focus.

\section{Mapping Institutional Progress with Learning Analytics}

Irrespective of definitions, the implementation of learning analytics in the sector is at an early stage (Colvin, et al., in press; Fiadhi, 2014; Johnson, Adams Becker, Estrada, \& Freeman, 2015; Sclater, 2014; West et al., in press). While a variety of case studies in relation to initiatives is available internationally (see Arnold \& Pistilli, 2012; Sclater, 2014; Yeado, Haycock, Johnstone, \& Chaplot, 2014), these tend to be of a relatively small scale, localised or narrow focus. They are useful to demonstrate what is possible and to share practice, but do not necessarily move others forward on implementation. Efforts to assist in this regard have tended to focus on frameworks for building organisational capacity and/or benchmarking more broadly, and related to technology enabled learning, as well as subsequent application of these to learning analytics. These have included a variety of foci including measuring capability (Marshall and Mitchell, 2002; Marshall, 2006; ACODE, 2014), advocating process (Bichsel, 2012; Campbell and Oblinger, 2007) and/or identifying domains for attention (Arnold, Lonn, \& Pistilli, 2014; Arnold, Lynch, et al., 2014; Sclater, 2014). Thus, most frameworks generally incorporate each of these, but it is useful to highlight some of the main elements. 
Benchmarking is a critical element of quality control mechanisms and as such the sector often looks for ways to assess our development in key areas. Much work has been done on this element related to technology enabled learning, including the earlier work of Marshall \& Mitchell (2002), and subsequently Marshall (2006). Their e-learning Maturity Model (eMM) is intended to assist institutions to deliver e-learning in a sustainable and high quality manner. Building on Chickering and Gamson (1987) they initially presented a model based on levels of capability. However, the second version removed the concept of levels, acknowledging that it implied a hierarchical structure which was unhelpful in a dynamic environment and they moved to one based on dimensions. The overall premise is that there needs to be capability across the dimensions which include: learning; development; support; evaluation and quality control; institutional planning and management. The concept of process around these is overlaid in each dimension. Each dimension then includes a list of statements to prompt reflection and rating. At the core, the model provides a way of assessing how an institution rates in relation to each dimension, from fully adequate to not adequate.

Similar to Marshall (2006), Educause's ECAR model (which is specifically focussed on learning analytics) attempts to provide a framework for measuring capability but places more emphasis on the domains. The domains included in this model are: process, culture, expertise, investment, governance/infrastructure and data/reporting tools. In a similar vein, The Learning Analytics Readiness Instrument (LARI) (Arnold, Lonn, et al., 2014) focusses on 5 domains: ability; data; culture and process; governance and infrastructure; and overall readiness perceptions. It is intended for use by institutions to develop an understanding of both their strengths and areas for development via rating against statements in each domain.

The use of a series of statements to rate one's progress is the hallmark of benchmarking frameworks. The Australian Council on Open and Distance Education (ACODE) outlines and follows this process in the development of their document for benchmarking technology enhanced learning. The document (ACODE, 2014) includes a scoping statement, good practice statement and performance indicators and measures; it also has room for rationale, evidence, and recommendations for eight domains. These domains include governance; planning; information technology (IT) infrastructure; application of technology; staff development; staff support; student training and student support. It is designed as a tool to be used actively by institutions for self-assessment and comparison.

Another version that focussed more specifically on learning analytics, and that incorporates stages and domains, is put forward by Norris and Baer (2013). Their model incorporates the domains of technology infrastructure; processes and practices; culture and behaviour; skills and values; and leadership. It includes three stages of capacity building from getting started to accelerating progress, and lastly to transforming/optimising analytics.

While most frameworks place some emphasis on process, for some this is a central focus; most notably Bichsel (2012) and Campbell and Oblinger (2007). Campbell and Oblinger identify 5 steps, which are: capture; report; predict; act and refine. Bichsel notes that while there is a wide degree of variation in how analytics might be used, it can be conceptualised as having a common cyclical process with the following five stages that loop back as refinement takes place:

1. Identify a strategic question

2. Find or collect relevant data to the question

3. Analyse the data with a view to prediction and insight

4. Form and present the data in understandable and actionable ways

5. Feedback into the process of addressing the strategic question and identifying new ones 


\section{Lessons from the Literature}

To conclude this brief overview, it is useful to highlight the themes that arise from the available frameworks. They all advocate, to a greater or lesser extent, the need for ongoing refinement and improvement via a process of reflection. They draw attention to the fact that development and progress in learning analytics is a complex, interdependent and dynamic endeavour, which encompasses a variety of stakeholders across the institution. Related to this, a variety of domains need to be attended to, including those most commonly noted: technical infrastructure, policy and governance, skills; support and culture. Implied within these frameworks, and explicit in the retention literature, is the need to undertake development in a way that is appropriate to any given institution. So while it is critical to share examples and strategies, there is a need to work more effectively within an institution to make clear progress. Working from these key ideas, our project sought to develop a framework for moving forward on the use of learning analytics for student retention.

\section{Project Context and Research Questions}

As learning analytics is at an early stage and considerable variety exists amongst analytics methods and products, and the individual contexts in which learning analytics might be deployed, it was difficult to hypothesise which themes would emerge most strongly over the course of the study. The project team was aware through its own professional networks and involvement in various forums that many universities were actively exploring and testing different tools, but that widespread and mature usage seemed fairly limited. Consequently, the project adopted a broad and exploratory scope with the intention to elicit information from a broad range of contexts. This is reflected by the two main research questions:

1. What factors are relevant or need to be considered where the implementation of learning analytics for student retention purposes is concerned?

2. How do these factors impact on the implementation of learning analytics for student retention purposes?

\section{Method}

Given that this paper is primarily focused on introducing a framework, it is useful to think of the method as having two distinct phases. The first is the initial mixed-method (survey and interview) data collection that informed the development of a draft framework. The second phase represents the National Forum where the framework was presented to peers for feedback and a process of refinement and adjustment that followed.

\section{Phase 1}

Institution level survey. The institution level survey, which can be viewed here, was conducted in July and August 2014, and was directed toward senior academic leaders who could give an overview of the institutional strategy around learning analytics. The survey was intended to help build a picture of sector progress with learning analytics more broadly and in relation to student retention, as well as data infrastructure, human resources, policy development and other elements that relate to learning analytics as identified through the literature. It was distributed to Deputy Vice Chancellors (Academic) (DVCA's) at each Australian university via email and promoted via the Universities Australia DVCA forum. Twentytwo Australian institutions participated. The sample was extended to New Zealand (NZ) following a request from the project reference group which resulted in two NZ institutions participating. The survey was built and hosted using the online Qualtrics application and was set to allow anonymous responses. 
SPSS was used for the quantitative analysis to produce descriptive statistics while manual coding was used to analyse qualitative questions.

Academic level survey. The academic level survey, which can be viewed here, conducted between September and November, 2014, was targeted at academic staff (e.g. teachers, student support, academic developers etc.), and focused on how they were thinking about and/or using learning analytics in the context of student retention. The survey employed a purposive, snowball sampling strategy to recruit self-selecting individuals $(n=353)$, whose responses were anonymous, with invitations circulated via existing networks, professional associations and conferences. Analysis involved a range of techniques including frequencies, means testing and tests of significance as appropriate to the individual quantitative questions and manual coding of themes for qualitative questions.

Table 1: Frequency distributions of demographic data about academic level survey participants

\begin{tabular}{|llrr|}
\hline Variable & Category & $\begin{array}{r}\text { Absolute } \\
\text { Frequency }\end{array}$ & $\begin{array}{r}\text { Relative } \\
\text { Frequency }\end{array}$ \\
\hline Location & & 341 & $97 \%$ \\
$\mathrm{n}=351)$ & Australia & 10 & $3 \%$ \\
\hline Primary Work Role & New Zealand & 188 & $53 \%$ \\
$(\mathrm{n}=353)$ & Teaching Students & 47 & $13 \%$ \\
& Learning Support & 37 & $11 \%$ \\
& Management/Administration & 32 & $9 \%$ \\
& Other & 24 & $7 \%$ \\
& Research & 18 & $5 \%$ \\
& Academic Development & 7 & $2 \%$ \\
\hline LMS at Institution & Student Support & 203 & $58 \%$ \\
(n= 353) & Blackboard & 124 & $35 \%$ \\
& Moodle & 13 & $4 \%$ \\
& Brightspace (D2L) & 2 & $1 \%$ \\
& Sakai & 11 & $3 \%$ \\
\hline Academic Level & Other & 30 & $9 \%$ \\
(n=351) & Associate Lecturer/Tutor & 124 & $35 \%$ \\
& Lecturer & 88 & $25 \%$ \\
& Senior Lecturer & 30 & $9 \%$ \\
& Associate Professor & 20 & $6 \%$ \\
& Professor & 59 & $17 \%$ \\
\hline Length of employment in Higher & Less than 1.5 years & 11 & $3 \%$ \\
Education Sector & $1.5-5$ years & 42 & $12 \%$ \\
(n= 345) & $5-10$ years & 85 & $25 \%$ \\
& $10-20$ years & 130 & $38 \%$ \\
& More than 20 years & 77 & $22 \%$ \\
\hline Involvement in teaching students & Teaches students & 276 & $78 \%$ \\
& Does not teach students & 77 & $22 \%$ \\
\hline
\end{tabular}

Table 1 presents a summary of sample demographics in the form of frequency distributions for a range of employment related indicators. While the sample represents a small proportion of the overall population of Australian academic staff potentially involved with student retention, it produced strong heterogeneity as far as teaching modalities, experience, location and role are concerned. 
Interviews. A series of semi-structured interviews were conducted between December 2014 and February 2015 with self-selecting participants who completed the academic level survey. The purpose of the interviews was to explore experiences with some of the key themes and issues uncovered in the quantitative elements of the project. Interviews were conducted with 23 people from 15 different universities. Participants held a variety of roles (e.g. teacher, educational developer, student support officer, librarian, learning analytics project leader, tutor, and learning and teaching leader) and spanned different academic levels. Each interview was digitally recorded (audio) and then transcribed verbatim for coding and utilisation in the framework development phase. Data from the interviews was thematically analysed using manual coding by two researchers.

Triangulation Process. The two surveys were designed to include question blocks which were complementary to provide data on key themes emerging from the literature from different stakeholder perspectives. Data from these two sources were brought together to identify where further information was needed which informed the interview questions. Thematic analysis of the interviews was then brought together with the data from the two surveys to allow for manual comparison of the findings from each, identification of trends and key concerns. This was undertaken using a discursive approach by four team members. Through this process the headline findings were identified with data collated under each. Throughout this iterative process, themes, concerns, tensions and areas of focus were recorded to feed into the development of the framework and the associated question sets.

\section{Phase 2}

The framework and the associated question set were developed by the project team as a graphic representation of the key elements identified through phase 1 of the project. The first version was presented to the project reference group and their feedback incorporated. In the meantime, the project partners utilised the framework to develop case studies and tested it as a tool to inform their own institutional thinking.

The next step was to present the framework at a National Forum for feedback. This took place in April 2015, where 148 colleagues from 43 different institutions came together to discuss the findings, explore the framework and share examples from their own experiences with learning analytics. The project had a budget that allowed each Australian institution to send one fully supported (transport and accommodation) representative to the forum. Typically, this person was someone involved in implementing learning analytics, or who would be involved if learning analytics were to be implemented at their institution (e.g. DVC Academic, Director of Learning and Teaching, and Business Intelligence leaders). The rest of the participants made their own way to the forum, though registration was free. This resulted in a further group of stakeholders that included teaching staff, educational designers and developers and researchers, among others.

The morning session of the forum focused firstly on broad presentation of the project findings followed by a specific introduction to the framework (for details of the program and presentations see West et al, 2015a). The afternoon session split participants into two smaller structured workshop groups to explore and test the clarity and usefulness of the framework in the context of participants' own institutions and roles. During these workshops participants worked in small groups and members of the project team recorded key issues raised. Following the forum, the project team reconvened and amended the framework with further advice being provided by the project reference group. 


\section{Results}

The results that follow are presented in two parts. The first of these is a series of 'headline' findings that spanned the three Phase 1 data sets (institutional level survey, academic survey and interviews). Given space limitations it is impossible to present all of the data tables and graphs but in this instance the headline findings are intended to lead into the presentation of the framework, which constitutes the second part of the results section. Readers interested in further exploring the data sets and findings can find this information on the project website (West et al, 2015a)

\section{Headline Findings}

Headline finding 1: The sector was at an early stage of development, implementation and understanding around learning analytics. Across the institution level survey, academic level survey and interviews, the data indicated that whilst pockets of learning analytics use existed in a number of institutions, there were many academics with very little awareness or participation in analytics discussions and projects. As an example, Figure 1 presents data from the academic level survey that illustrates this point. It shows results when participants were asked how often they discussed learning analytics with colleagues in different groups, with the darker bars on the right of each series showing less frequent discussion.

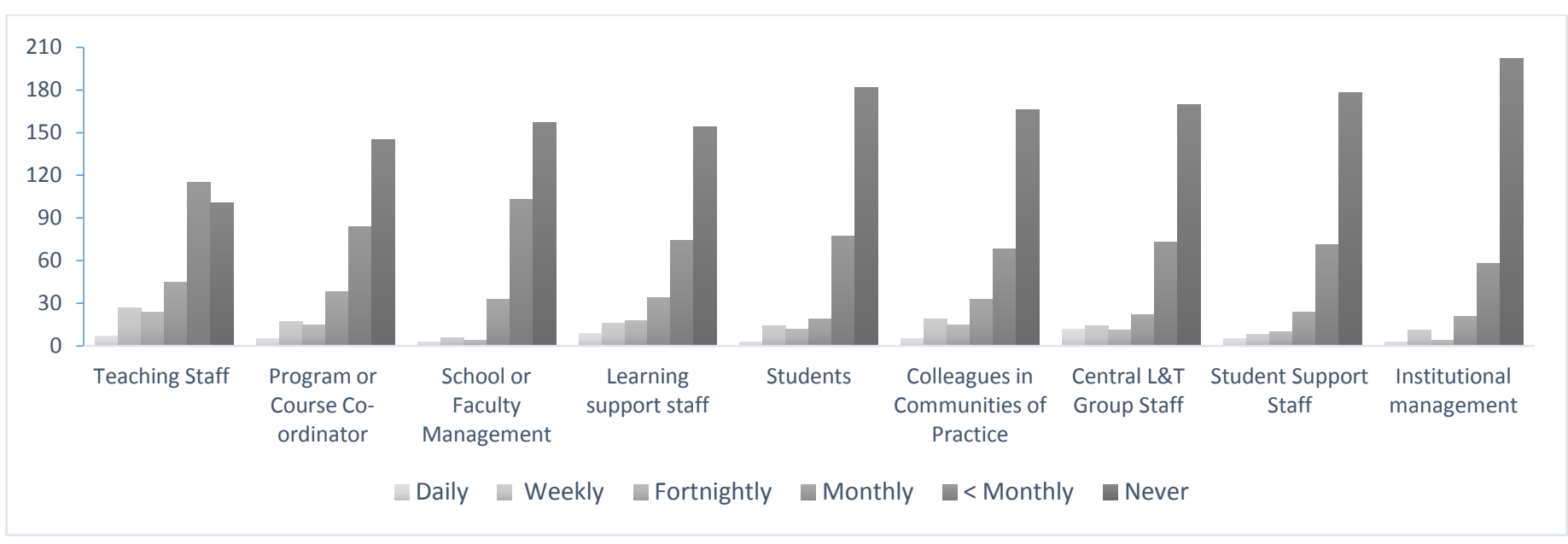

Figure 1: Frequency of discussion that participants reported with selected colleagues

The complexity of learning analytics may mean that development will take time and this may limit opportunities for academics to be involved and develop confidence with learning analytics in the short term. As one interviewee involved in learning analytics implementation stated: "I guess I am looking at where we might end up focusing on the spectrum of learning analytics and I don't really have an answer for that just yet”. This is one quote that was indicative of many similar responses.

Headline finding 2: There was a degree of cautiousness reflecting an emerging recognition of the amount of thinking that needs to go into how learning analytics can be applied. Following on from the first headline finding, it became clear that perceptions differed on what learning analytics implementation might look like, as the following interview quote suggests:

At our university, we're not too far advanced. We don't have that kind of system integration yet, that allows us to do that. Before we go headlong into assuming that it is all about system integration and spending huge amounts of money on trying to make systems talk to each other 
that don't want to, that if we drive the conversation on the basis of understanding what are the key points of data, it might be that those systems don't actually necessarily need to talk to each other, but we might be able to aggregate that information at another level.

This is where variation in leadership and strategic thinking can impact on what gets implemented and when. In some institutions there was a clear expression by those setting the direction that getting data into integrated systems, such as a data warehouses, would create the flexibility to address questions as they arise - one did not necessarily need to know what those questions were at the time of building the data warehouse. In contrast, other participants' views were more indicative of the quote above, which reflects a problem by problem approach in which implementation is driven by clearly identified needs and problems.

Headline finding 3: There was great variability across the sector - in relation to preparedness, how institutions are thinking about and implementing learning analytics due to context. The first two headline findings illustrate the complexity and broad scope of learning analytics and how this complexity posed challenges for participants in the study. The third headline finding follows logically but speaks to a different kind of complexity, which is the significant variation that can occur across institutional contexts and environments. This can take many forms, for example, variation in organisational culture as articulated by one interviewee:

I think the real learning for me so far has been the need for the cultural environment at the institution and the associated drivers and leadership. The data has to have a certain environment or a certain context before it has any meaning whatsoever. That way you can clearly mark what that is and you can find a platform for it to have a sort of life within the environment.

Another commonly expressed example of variation in institutional context related to the student cohort. One participant described the student cohort at their university as "fairly competitive", another spoke of a large population of "low socio-economic" students, whilst another again spoke of their university being very "research focused". Taken together, these examples point to the importance of institutional context in shaping how learning analytics might be implemented and the framework unpacks this further.

Headline finding 4: Participants identified tensions and questions around the extent to which learning analytics can drive actions and behaviours or take the functions of people. Perhaps not surprisingly, given the Figure 1 data that illustrated the limited amount of analytics focused discussion taking place involving faculty, there was some uncertainty about how students would experience learning analytics, particularly automated communication. This tension was captured by one participant, who observed:

Even though you are using automated systems to communicate and provide feedback you still have to make sure that it is personalised and meaningful for students and that takes time and consideration...In the system you can use this code so you don't have to write names every time and you don't have to use hello everyone all the time. This gets more attention from students and encourages students to be engaged.

Another participant offered the view that there would still be a major role for direct person-toperson communication with students.

Statistics can be helpful, but it can also be useful to talk to students directly and see what they actually think they need themselves. From all of this we can work on programs, strategies and supports that might help them stick around a bit longer. 
Whilst these two quotes express a consciousness of student needs they also potentially allude to a broader fear that one’s job or work role might be impacted on, or even superseded, by computers.

Headline finding 5: There were substantial gaps between academics' needs and aspirations and what is being provided, what is available, and what is being developed. One of the challenges the sector faces in widely leveraging the potential of learning analytics is how to distribute knowledge and awareness of what can be achieved through learning analytics. As the previous quotes illustrated there are both real and potential fears around the implications of learning analytics for academic staff, particularly where academics are not well informed about what learning analytics are, how their institution plans to use them, and how they are expected to use them. The data in Figure 2 reinforces this point. Taken from the academic level survey, it shows that a large proportion of participants rated their institution as 'poor' or 'very poor' at meeting their needs in relation to some basic learning analytics provisions. Further illustrating the uncertainty felt by participants is that a relatively high proportion were unsure how they would rate their institution $-n$ for 'not sure' varied between 85 and 104 across the six variables.

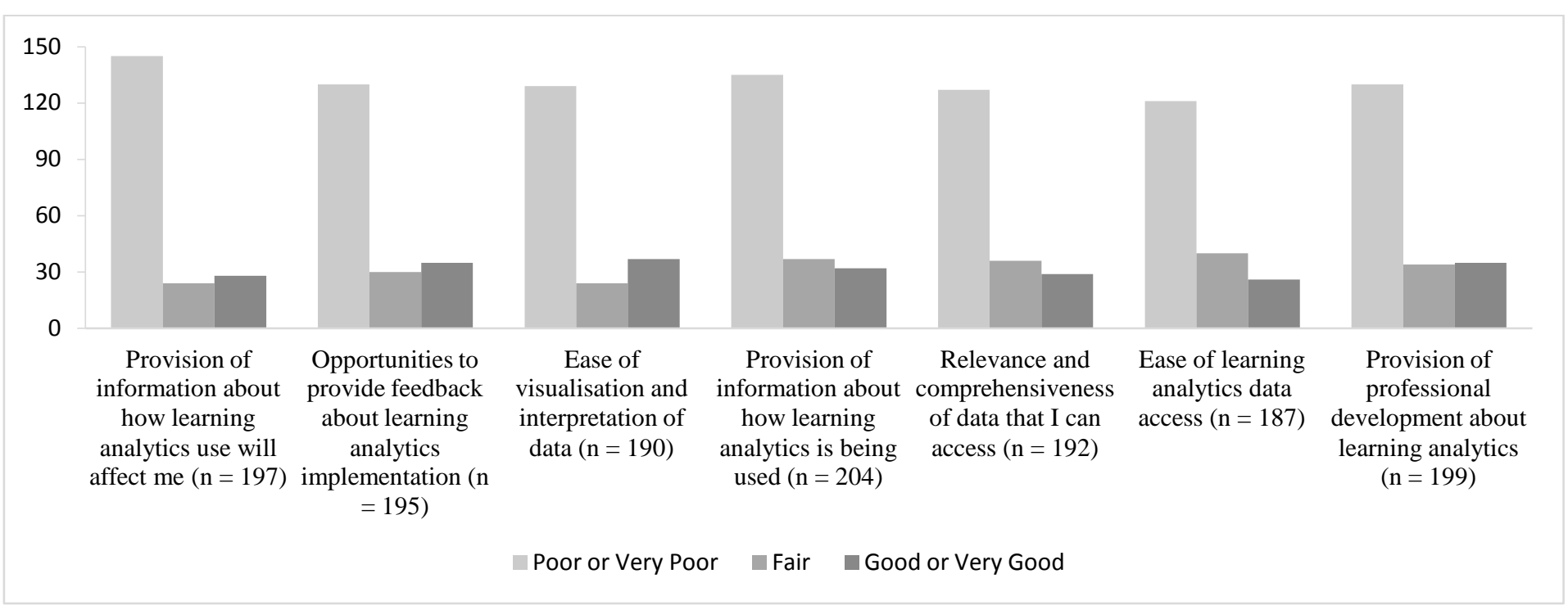

Figure 2: Participants rating of their institution at meeting their needs around selected learning analytic provisions (excludes ‘not sure’ responses to show trends visually)

The messages in this data are consistent with data provided by a number of interviewees as well as the institution level survey, which suggested many institutions were currently engaged in localised pilot projects and/or institution level systems development, which for various reasons may not be well publicised to the wider staff cohort in both cases. This leads to the final headline finding.

Headline finding 6: There was tension between business needs, wants and limitations (e.g. costs) versus academic staff needs and wants (e.g. academic freedom, innovation in learning and teaching). A recurring theme across the two surveys and interviews was that the roles of individual staff and work groups are a key factor in determining the types of learning analytics applications they might be interested in, and to some extent this is where the ambiguity around educational and learning analytics has impacts. There are two parts to this.

The first is that a number of participants expressed a desire for analytics to help inform their work, but there was also an expressed desire for academic freedom i.e. not being told how to teach, what forms grades had to be entered, or when to commence assessment tasks, for example. This can be 
problematic when particular processes need to be followed in order that data is cleanly and consistently recorded and thus usable. The second part relates to the beneficiaries of learning analytics. There are questions about the data that might be used for learning analytics, especially who owns it and how it can and should be used. When participants were asked about the ethical principles that should guide learning analytics, a number talked about the highest priority being student benefit. Others went as far as saying that the institution should not be using learning analytics for its own benefit.

\section{Linking the Data and the Literature: A Summary of Lessons}

These headline findings and a more granular thematic analysis of survey and interview responses (West et al, 2015) led the project team to consider how the various frameworks/models and benchmarking documents assist in implementation and address the issues described by participants. Key lessons from this reflection included:

- Despite a range of examples of learning analytics initiatives being available, people in the sector are struggling with institution level implementation of learning analytics, especially in terms of aligning policies, systems, people and stakeholder needs.

- Institutional context will change the shape of development and pathways for implementation

- A reflective, non-linear model is essential

- $\quad$ The domains outlined in the literature are essential but not sufficient, particularly in trying to address student success

- $\quad$ The models/frameworks are more broadly about technology enabled learning and/or learning analytics but are not focussed on the issue of student success

- Currently, there is a lack of clear alignment between the work of researchers, institutional learning analytics leaders and academic staff, particularly in terms of working together on problems of agreed strategic importance. The data in Figure 1 showed that academics in this sample were engaging in a relatively limited amount of dialogue with other colleagues, making strengthening dialogue a logical starting point.

\section{Introducing the Let's Talk Learning Analytics Framework}

With these assumptions in mind, the Let's Talk Learning Analytics Framework was developed (and refined as per the methodology above). It consists of two primary documents:

1. A one-page high level summary of the framework (Figure 3), which consists of six key domains. The six domains, shown in green, are the areas an institution needs to consider to make progress on learning analytics for retention. They are interconnected and shaped by the primary domain, the institutional context. Continual reflection and adjustment are critical to the process and will require input from across the institution.

2. A set of discussion questions (West et al, 2015c) to support the use of the framework, organised around the key domains. The purpose of the discussion questions is to provide a series of prompts to guide a contextualised discussion and is based on the premise that dialogue across an institution is critical.

The set of questions which accompany the framework are essential but, for this paper, will be split and presented under each of the domains to help illustrate the relevance of those domains. In practice it would be expected that learning analytics leaders would use the questions and framework documents side-by-side in a meeting, workshop, or any other environment centered on dialogue, or a combination of these in line with the yellow section in Figure 3. The questions are not statements related to stages or levels but rather, prompts, to engage in discussion and reflection to ensure that key elements are 
articulated, clarified or marked for development. The framework is also seen as dynamic and non-linear with continual reflection and adjustment, based on the idea of transitioning to learning analytics use. The term 'transitional' is used to reflect that learning analytics is an emerging field and many institutions will be transitioning to an environment that, for the first time, broadly incorporates learning analytics (e.g. into institutional policies and plans), as well as transitioning to an environment where learning analytics is specifically applied to issues like student retention. As such there is no implied point for starting the process beyond the institutional context. There is an expectation that institutions will engage in a process of praxis (reflection, action, reflection).

\section{INSTITUTIONAL CONTEXT}

Institutional context provides the parameters for what is feasible/appropriate and includes:

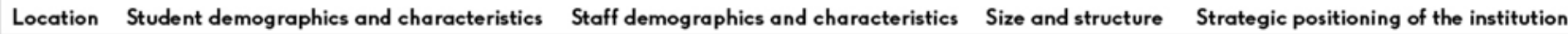

\section{SYSTEMATIC DISCUSSION OF THE SIX DOMAINS - GUIDED BY THE DISCUSSION QUESTIONS RESOURCE}

This discussion interface recognises that sucessfully coping with the complexity of learning analytics requires a collaborative, systematic approach because: Expertise relating to the six different domains is distributed across institutions Learning analytics projects typically exert broad impact across an institution

\begin{tabular}{|c|c|c|c|c|}
\hline $\begin{array}{l}\text { TRANSITIONAL INSTITUTIONAL } \\
\text { ELEMENTS }\end{array}$ & $\begin{array}{l}\text { LEARNING ANALYTICS } \\
\text { INFRASTRUCTURE }\end{array}$ & $\begin{array}{l}\text { TRANSITIONAL RETENTION } \\
\text { ELEMENTS }\end{array}$ & $\begin{array}{l}\text { LEARNING ANALYTICS } \\
\text { FOR RETENTION }\end{array}$ & $\begin{array}{l}\text { INTERVENTION AND } \\
\text { REFLECTION }\end{array}$ \\
\hline $\begin{array}{l}\text { Transitional institutional } \\
\text { elements provide the } \\
\text { parameters for the } \\
\text { implementation of learning } \\
\text { analytics. They include: } \\
\text { Culture } \\
\text { Positioning of learning } \\
\text { analytics within the } \\
\text { institution } \\
\text { Level of sponsorship } \\
\text { Governance arrangements } \\
\text { Alignment with institutional } \\
\text { strategy } \\
\text { Sustainability }\end{array}$ & $\begin{array}{l}\text { Learning analytics } \\
\text { infrastructure is concerned } \\
\text { with three main factors: system } \\
\text { reliability, system } \\
\text { sophistication and relevant } \\
\text { expertise. This includes: } \\
\text { Digital availability and } \\
\text { integrity of data } \\
\text { Integration, continuity and } \\
\text { availability of data systems } \\
\text { Technical, pedagogical, } \\
\text { statistics, and project } \\
\text { management expertise } \\
\text { Data stewardship } \\
\text { Policy and procedures }\end{array}$ & $\begin{array}{l}\text { Transitional retention elements } \\
\text { provide the parameters to } \\
\text { enable more effective } \\
\text { deployment of learning } \\
\text { analytics for retention } \\
\text { purposes. These include: } \\
\text { Retention planning } \\
\text { Retention strategy and } \\
\text { implementation } \\
\text { Governance arrangements } \\
\text { related to student retention }\end{array}$ & $\begin{array}{l}\text { Learning analytics for } \\
\text { retention factors are focussed } \\
\text { on the use of learning analytics } \\
\text { for retention and include: } \\
\text { Educational and business } \\
\text { questions from various } \\
\text { stakeholders } \\
\text { The ability of the system to } \\
\text { address the questions } \\
\text { Accessibility, ease of use of } \\
\text { system, tools and reports for } \\
\text { various stakeholders } \\
\text { Consideration and resolution } \\
\text { of the ethical issues which } \\
\text { may arise from the } \\
\text { implementation and use of } \\
\text { learning analytics }\end{array}$ & $\begin{array}{l}\text { Intervention and reflection } \\
\text { are critical to improving } \\
\text { retention. Consideration } \\
\text { needs to be given to: } \\
\text { Training, support and time } \\
\text { for staff and students to use } \\
\text { systems, interpret data and } \\
\text { reports and act on them } \\
\text { Endorsed processes around } \\
\text { actions or interventions } \\
\text { arising from the data } \\
\text { Modification of relevant } \\
\text { elements, systems, factors } \\
\text { and interventions }\end{array}$ \\
\hline
\end{tabular}

Figure 3: Let’s Talk Learning Analytics Framework

Domain 1: Institutional Context. The central message from the data was that institutional context is the guiding feature of analytics implementation. It sets the scene for all of the decisions that will follow and as such is seen as foundational and therefore positioned in the framework across the top to highlight the fact that it will pervade all other domains. The questions for consideration in this domain include:

- What are the implications of the jurisdictional location of your institution (e.g. privacy laws)?

- What is the size and structure of your institution?

- What is the strategic positioning of your institution (e.g. partnerships, international, and areas of focus)?

- What are the student demographics and characteristics?

- What are the staff demographics and characteristics? 
Domain 2: Transitional Institutional Elements. Transitional elements in this framework are used as a term to both narrow the focus and to suggest that the elements that sit in these domains are a lens by which to view other domains and elements. They will inevitably shape the development of learning analytics development in an institution. For example, in institutional transitions, the executive commitment and position is critical to progress as it will impact on investment, practices and culture (see Arnold et al, 2014 and Norris \& Baer, 2012). Key questions for discussion in this domain include:

- How does the culture at your institution both promote and challenge the advancement of learning analytics?

- What is the strategic positioning of learning analytics within your institution?

- To what degree is there executive sponsorship (academic and IT)?

- Do you have a specific learning analytics plan and if so, how is it aligned with your institutional budget and academic plan?

- To what extent is there congruence between the vision, strategy and values of the institution that relate to learning analytics?

- How clearly is the role of learning analytics in your institution identified and communicated?

- How sustainable is the approach to learning analytics at your institution?

Domain 3: Learning Analytics Infrastructure. The Learning Analytics Infrastructure domain is a representation of the importance of data systems and infrastructure to the use of learning analytics. There is no doubt that institutions have been collecting and storing significant amounts of data for years (Educause, 2010; Heathcote \& Dawson, 2005). However, these have not necessarily been accessible in the right format or able to be used effectively. Project participants cited numerous examples of issues around data and systems where the interface is not user-friendly, the data is incomplete, data integration is inadequate, or the time taken to obtain a report or visualisation is too long.

This domain includes three key questions which are then subsequently broken down into more specific questions under the headings of digital availability of data, data integrity, systems continuity, availability and reliability, integration of data systems, expertise, stewardship and data policy and procedures. For our purposes only the three key questions are presented here (the fuller set area available on the project website (West, 2015c).

- $\quad$ Are your systems reliable?

- Are they technically sophisticated enough?

- Is there enough expertise in the institution?

Domain 4: Transitional Retention Elements. As this framework is specifically focussed on the use of learning analytics for student retention, this domain draws attention to that lens and transitions us from thinking about all the things learning analytics might be used for, to how it can be used to support student success. Key questions in this transition domain include:

- Do you have an institutional retention plan or strategy?

- What are the governance arrangements that support the retention plan or strategy?

- How are learning analytics positioned within the retention plans and associated governance arrangements?

It is important to reiterate that this framework was developed for a project with a specific focus on student retention. This explains the focus of Domains 4 and 5. It is possible to adapt these domains to other key foci by changing the focus of Domains 4 and 5, whilst keeping the other domains as they are. 
Domain 5: Learning Analytics for Retention. This domain highlights key questions that prompt thinking around the more specific area of learning analytics for retention (rather than business intelligence, action analytics etc.) and so should be considered in that light.

- What business and educational questions do your stakeholders have, and how are they prioritised?

- To what extent can your system address those questions?

- Where gaps are identified, what resources are available for follow up?

- What ethical issues have been identified and what is the resolution?

Domain 6: Intervention and Reflection. Data gathered throughout the project demonstrated that there is a wide range of learning analytics activities taking place throughout the sector. However, many of these are small scale or pilot projects are not necessarily centrally led and take place in various locations with different intended outcomes. Yet, projects with no action will have little impact on student success and they thus need to be connected to some sort of intervention (whether the data informs an action (utilising people to call students) or be part of the action (e.g. automated messages to students or connections to personalised learning). Intervention at this stage also includes things like training programs for staff and students, which was clearly identified as a gap in the data.

It was also clear that while reflection is necessary in an ongoing manner, it needed to be more explicit in this area to ensure that lessons from learning analytics projects are fed back in to other work. A clear example of this might be that a learning analytics project demonstrates that a particular strategy in the retention plan is not effective. This should prompt investigation and subsequent revision of the retention plan. Key questions in this domain include:

- How are potential interventions developed, managed and enabled?

- What training is provided, and what training is required for staff and students?

- What time and support is provided, and what time and support are required, for staff and students?

- How are the interventions evaluated and improved?

- How is the whole system evaluated and improved?

\section{Discussion}

While this framework builds on prior work (Arnold, Lonn, et al., 2014; Bischsel, 2012; Norris \& Baer, 2012), it has some key differences. These include an emphasis on dialogue, a focus on the use of learning analytics for student retention and success, and placing an explicit focus on both the foundation of context and the action/intervention required. It attempts to draw attention to the inter-related elements that will take learning analytics in different, but equally useful directions.

Clearly the level of maturity and capacity that institutions have when it comes to learning analytics varies and though there has been much research interest in learning analytics, this is not necessarily translating into coherent, widely understood plans and strategies within institutions. The flowon effect of this is that academic staff may be anxious about falling behind with a new set of tools (i.e. learning analytics) that they keep hearing about. Additionally, they may not be aware that their knowledge and skills could be harnessed in relation to institutional learning analytics work. In this way, the framework is seen as a key tool for creating constructive dialogue around the potentially daunting changes that learning analytics bring. 


\section{The Role of Dialogue}

The emphasis on dialogue hinges on several factors that were very evident in both the literature and the data. First, that learning analytics is complex and in a nascent stage of development, which means that we are still exploring the potential that it affords. In order to explore and leverage the potential, we need to have the involvement of people from multiple disciplines and backgrounds (Balacheff \& Lund, 2013; Siemens, Dawson \& Lynch, 2013) (but particularly IT and pedagogy), and points of view (such as teaching academics, head of schools, senior executive and students) at every stage. This includes planning, development, deployment, and feedback. Yet the data suggested that such knowledge and input was largely occurring in fairly narrow silos or select groups, and teaching academics in particular were mostly unaware of the initiatives taking place in their own institutions. As noted in Figure 1, most academics in the survey very rarely had discussions with anyone around learning analytics. This is not particularly surprising given that many initiatives were small pilot projects of limited scope. Additionally, the complexity of learning analytics initiatives from a technical view has placed it largely in the realm of IT departments or learning analytics specialists. Breaking down the potential silos is critical and dialogue is one way to do this.

Furthermore, many people (even at higher levels) in the sector have had limited practical exposure to learning analytics and therefore do not always recognise tangible implications that impact on implementation. Finally, learning analytics implementation necessitates collaboration between people with vastly different roles and expertise. For example, the use of even basic reporting for functions from software packages which integrate the LMS and SIS, need a level of standardisation in practice to be effective. Items such as the grade centre need to be set up in an appropriate way in order to display some of the reports. Conversations about these factors and which reports would be most useful from the perspective of the teaching academic, could help in a range of ways including to prioritise work in analytics implementation; aid in a level of standardised practice by teaching academics; and, link to support mechanisms for staff and students.

Bichsel (Bischsel, 2012) recommends that institutions use a maturity index (specifically the Educause maturity index) to gauge analytics progression, set goals and measure progress. While this type of benchmarking is useful, the idea of plotting progression on a scale is generally of interest to particular stakeholders. Additionally, it is not designed to be an implementation tool or guide but it is about measuring progress instead. In contrast the framework presented here is not about benchmarking but about providing a set of discussion questions to be actively used to move implementation (or at least dialogue on analytics) forward. This is very much in line with the suggestions of Bichsel (2012) who recommends attention to planning, process and partnership across various stakeholders.

\section{Analytics for Student Retention and Success}

As noted at the outset of this paper, the area of student retention and success is a high priority in the sector. This focus has flowed through to the use of learning analytics being harnessed to assist in this area (for example see (Arnold \& Pistilli, 2012; Sclater, 2014; Yeado et al., 2014). Yet frameworks and benchmarking tools on analytics are about analytics more broadly, which, while useful, does not prompt stakeholders to think about the complex area of student retention nor to frame the work around the broader context of student success. While perhaps there is an assumption that that we would be using learning analytics to improve the student journey, there is value in making it more explicit.

While the authors acknowledge and support the idea that learning analytics can be used for a wide range of purposes, central to this is the idea of student retention and success. This project was funded to consider learning analytics in light of retention but it was very apparent that thinking in the sector has moved to the broader construct of student success with retention being a subset of that. In many ways a 
clear focus on the student journey may help to move the learning analytics journey forward. As Siemens (2012: 4) observes: "A transition in analytics from a technical orientation to one that emphasizes sensemaking, decision-making, and action is required to increase interest among educators and administrators. This transition in no way minimizes the technical roots of analytics; instead, it recasts the discussion to target problems that are relevant to practitioners instead of researchers". It is perhaps the student journey which may be able to provide a point of reference for engagement of a more diverse set of stakeholders.

The work in the area of student retention can also provide some guidance in terms of the types of initiatives which may be useful, and this will flow through to the types of data that would be collected, the types of reports required, who should have access to those reports and the subsequent actions that might take place. By way of example, Transition Pedagogy (Kift, Nelson \& Clarke, 2010; Kift, 2009; Kift \& Nelson, 2005) focuses on the concepts of transition, diversity, design, engagement, assessment and evaluation as being critical to first year retention. If an institution was using such a framework it would be much clearer on how to focus learning analytics development. Additionally, some of the concepts within Transition Pedagogy are notoriously difficult to measure and learning analytics may offer some opportunities to revisit such work to both test and expand the model.

\section{Context}

Context is critical. This basic statement was a central theme of the data in terms of the initiatives occurring, what was possible, the resources and infrastructure available and the priorities set to name just a few. In this sense, case studies and examples of what has worked in other places are useful but not sufficient. A good example of context within a domain is the strategic positioning of learning analytics within an executive portfolio. In some institutions this sits within an academic portfolio and in others in the IT portfolio. Such position in one or the other is both a reflection of how the institution views learning analytics and its purpose, as well as a driving force for future direction. Starting with the context will ensure that the initiatives that take place are appropriate to that environment or at least considered in this light.

Leveraging analytics is a dynamic and interactive process. There are a range of implementation decisions which need to be made along the way. There is the potential for these to be driven by a narrow focus rather than an institutional one, potentially leading to a mismatch between the decision and the direction of the institution, or, if present, the retention plan. For example, the decision to turn on a student facing dashboard is deceptively simple but may require deeper consideration in some contexts. Consideration may need to be given to the support that is provided around this, the nature of the report or dashboard, and the type of follow-up that takes place. Additionally, such dashboards should be built in relation to key pedagogic or retention questions. It is essential that each one of these decisions is in line with the overall plan and approach, and seen in context. The point is that there is no simple right or wrong but rather decisions that are built in line with institutional elements.

Finally, the decision to pursue learning analytics is costly in terms of time, expertise and money. If the decision and subsequent implementation is done in a systematic way and includes various stakeholders and expertise in development, it is more likely to have an impact. A number of participants emphasised that inadequate communication, where learning analytics implementation is concerned, is liable to cost time and money. Therefore, from an efficiency point of view the use of a dialogical framework can prompt the communication in a focussed way and avoid unnecessary delays and align processes for outcomes. 
Let’s Talk Learning Analytics: A Framework for Implementation in Relation to Student Retention

\section{Limitations}

Prior to the concluding remarks some potential limitations are acknowledged:

- Learning analytics is rapidly developing area so it is likely that ongoing data collection is required to gather updated perceptions, experiences and aspiration from academics and institutions as they get more experience with learning analytics. To address this limitation, the project team plans to conduct follow up data collection in the coming period.

- $\quad$ The data used to develop the framework was from Australia and New Zealand, so readers will need to consider how this might impact on the applicability of the framework in their location, or other locations. However, because it is a discussion framework the researchers are interested in hearing feedback from peers in other locations and this will potentially assist in further refinement.

- The process of developing and refining the framework via data and various forums was highly iterative and describing the nuances of that method for the purposes of replicability is a challenge. Questions can be directed to the researchers.

- In terms of this specific paper it must be acknowledged that space restrictions also limited the amount of raw data that could be presented.

\section{Conclusion}

This paper has presented findings from a mixed-method study of Higher Education institutions and academics from Australia and New Zealand. Whilst this project produced a range of data on the perceptions, experiences and aspirations around learning analytics, one of the major aspirations of the project was to translate this data into a framework to promote institutional implementation bolstered by robust and systematic discussion. This has taken the form of the Let's Talk Learning Analytics Framework, and its presentation has been the primary focus of this paper. The framework can be thought of as comprising two complimentary tools. The first is the map of domains and factors relevant to institutional implementation of learning analytics and the second is a series of discussion questions that act as prompts within each of the domains.

Overall the framework is designed to foster a collegial approach to the implementation of learning analytics which is based on providing prompts for dialogue amongst key stakeholders. In this way it compliments existing frameworks and models which are more oriented toward measuring progress. Ultimately, it is hoped that this will assist in positioning learning analytics in a shared space which equally values pedagogical and technical expertise. Most importantly, it suggests that a focus on the student journey and the associated operational plans of an institution will be more likely to connect analytics to action for the benefits of students.

In the same way that it takes a community to raise a child, it takes an institutional community to truly make the most out of learning analytics. 


\section{Acknowledgements}

This project was funded by the Australian Government Office for Learning and Teaching. The views expressed in this publication do not necessarily represent those of the Australian Government Office for Learning and Teaching.

The authors would like to acknowledge that this framework was developed by the entire project team which included the authors and Professor A Lizzio, Griffith University; Professor C Miles, Newcastle University; Dr J Bronnimann, Batchelor Institute of Indigenous Education, Mr D Toohey, Murdoch University and Mr B Searle, Charles Darwin University.

\section{References}

ACODE. (2014). Benchmarks for Technology Enhanced Learning. Canberra, ACT: ACODE.

Arnold, K., Lonn, S. \& Pistilli, M. (2014). An exercise in institutional reflection: The Learning Analytics Readiness Instrument (LARI). Proceedings $5^{\text {th }}$ International Conference on Learning Analytics and Knowledge Conference (LAK'15), Poughkeepsie, New York.

Arnold, K., Lynch, G., Huston, D., Wong, L., Jom, L. \& Olsen, C. (2014). Building institutional capacities and competencies for systemic learning analytics initiatives. Proceedings Learning Analytics and Knowledge Conference (LAK'15), Poughkeepsie, New York.

Arnold, K. \& Pistilli, M. (2012). Course signals at Purdue: Using learning analytics to increase student success. Proceedings 2nd International Conference on Learning Analytics and Knowledge (LAK '12), Vancouver, British Columbia.

Balacheff, N. \& Lund, K. (2013). Multidisciplinarity vs. multivocality: The case of "Learning Analytics". Proceedings of the $3^{\text {rd }}$ International Conference on Learning Analytics and Knowledge (LAK '13), Leuven, Belgium. doi:10.1145/2460296.2460299

Behrendt, L., Larkin, S., Griew, R., \& Kelly, P. (2012). Review of higher education access and outcomes for Aboriginal and Torres Strait Islander People: Final report. Canberra: Australian Government.

Bichsel, J. (2012). Analytics in higher education: Benefits, barriers, progress and recommendations. Louisville, CO: EDUCAUSE Center for Applied Research.

Bradley, D., Noonan, P., Nugent, H. \& Scales, B. (2008). Review of Australian higher education: Final report. Canberra: Australian Government.

Buckingham Shum, S. \& Ferguson, R. (2012). Social learning analytics. Educational Technology \& Society, 15(3), 3-26.

Campbell, J., and Oblinger, D. (2007) Academic analytics: White paper. Educause. Retrieved 12 May, 2015 from http://net.educause.edu/ir/library/pdf/pub6101.pdf

Colvin, C., Rogers, T., Wade, A, Dawson, S., Gasevic, D., Buckingham Shum, S., Nelson, K., Alexander, S., Lockyer, L., Kennedy, G., Corrin, L., \& Fisher, J. (in press). Student retention and learning 
Let’s Talk Learning Analytics: A Framework for Implementation in Relation to Student Retention

analytics: a snapshot of Australian practices and a framework for advancement. Strawberry Hills, NSW: Office for Learning and Teaching.

Dekker, G., Pechenizkiy, M. and Vleeshouwers, J. (2009). Predicting students drop out: A case study. Proceedings 2nd International Conference on Educational Data Mining (EDM'09), pp. 41-50.

Educause (2010). 7 Things you should know about analytics. Retrieved May 12, 2015 from http://www.educause.edu/ir/library/pdf/ELI7059.pdf

Educause Center for Applied Research (ECAR) (2012). Analytics Maturity Index for Higher Education. Retrieved July 21, 2015 from http://www.educause.edu/ecar/research-publications/ecar-analyticsmaturity-index-higher-education

Ferguson, R. (2012). Learning analytics: drivers, developments and challenges. International Journal of Technology Enhanced Learning, 4(5/6), 304-317.

Fiaidhi, J. (2014). The next step for learning analytics. IT Pro, September/October, 4-8.

Frankola, K. (2002). Why online learners drop out. Retrieved 14 June 2013 http://www.kfrankola.com/Documents/Why\%20online\%20learners\%20drop\%20out Workforce. pdf

Gaševic, D., Dawson, S. \& Siemens, G. (2015). Let's not forget: Learning analytics are about learning. TechTrends: Linking Research and Practice to Improve Learning, 59(1), 64-71.

Heathcote, E. \& Dawson, S. (2005). Data mining for evaluation, benchmarking and reflective practice in a LMS. Proceedings e-Learn 2005: World conference on e-Learning in corporate, government, healthcare \& higher education, Vancouver, Canada.

Johnson, L., Adams Becker, S., Estrada, V. \& Freeman, A. (2015). NMC Horizon Report: 2015 Higher Education Edition. Austin, TX: New Media Consortium.

Kift, S. (2009). Articulating a transition pedagogy to scaffold and to enhance the first year student learning experience in Australian higher education. Strawberry Hills, ACT: Australian Government (ALTC).

Kift, S. \& Nelson, K. (2005). Beyond curriculum reform: Embedding the transition experience. In Brew, A. \& Asmar, C. (Eds.), Higher Education in a changing world: Research and Development in Higher Education (pp. 225-235). Milperra, NSW: HERDSA.

Kift, S., Nelson, K. \& Clarke, J. (2010). Transition Pedagogy: A third generation approach to FYE - A case study of policy and practice for the higher education sector. The International Journal of the First Year in Higher Education, 1(1), 1-20.

Koshy, P. (2014). Student equity performance in Australian higher education: 2007 to 2012. National Centre for Student Equity in Higher Education (NCSEHE), Perth: Curtin University.

Long, P. \& Siemens, G. (2011). Penetrating the fog: Analytics in learning and education. EDUCAUSE Review, 46(5), 30-32.

Marshall, S. (2006) eMM Version Two Process Guide. Wellington: Victoria University. 
Let’s Talk Learning Analytics: A Framework for Implementation in Relation to Student Retention

Marshall, S. \& Mitchell, G (2002). An E-Learning Maturity Model? Proceedings 19th Annual ascilite Conference, Auckland, New Zealand.

Nelson, K., Clarke, J., Stoodley, I. \& Creagh, T. (2014). Establishing a framework for transforming student engagement, success and retention in higher education institutions. Final Report 2014, Canberra: Australian Government Office for Learning \& Teaching.

Norris, D., \& Baer, L. (2012). A Toolkit for Building Organizational Capacity for Analytics (pp. 1-180). Washington, DC: Strategic Initiatives Inc.

Rivera, J., \& Rice, M. (2002). A comparison of student outcomes and satisfaction between traditional and web based course offerings. Online Journal of Distance Learning Administration, 5(3).

Sclater, N. (2014). Learning analytics: The current state of play in UK higher and further education. JISC, United Kingdom.

Siemens G. (2012). Learning analytics: Envisioning a research discipline and a domain of practice, Proceedings 2nd International Conference on Learning Analytics and Knowledge (LAK'12), Vancouver, British Columbia.

Siemens, G., Dawson, S. \& Lynch, G. (2013). Improving the productivity of the higher education sector: policy and strategy for systems-level deployment of learning analytics. Canberra, ACT: SoLAR/Australian Government Office for Learning and Teaching.

Universities Australia (2013). A smarter Australia: An agenda for Australian higher education 20132016. Canberra, ACT: Universities Australia.

van Barneveld, A., Arnold, K., \& Campbell, J. (2012). Analytics in higher education: Establishing a common language. Educause Learning Initiative. Retrieved May 10, 2015 from http://net.educause.edu/ir/library/pdf/eli3026.pdf

West, D., Huijser, H., Heath, D., Lizzio, A., Miles, C., Toohey, D., Brönnimann, J. \& Searle, B. (in press). Learning analytics: Assisting universities with student retention. Strawberry Hills, NSW: Australian Government Office for Learning and Teaching.

West, D., Huijser, H., Heath, D., Lizzio, A., Miles, C., Toohey, D., Brönnimann, J. \& Searle, B. (2015a). Forum resources. Retrieved September 25, 2015 from http://www.letstalklearninganalytics.edu.au/national-forum/

West, D., Huijser, H., Heath, D., Lizzio, A., Miles, C., Toohey, D., Brönnimann, J. \& Searle, B. (2015b). Discussion Questions. Retrieved September 25, 2015 from http://www.letstalklearninganalytics.edu.au/wp-content/uploads/2015/06/Discussion-QuestionsFinal-26-May-2015.pdf

West, D., Huijser, H., Heath, D., Lizzio, A., Miles, C., Toohey, D., Brönnimann, J. \& Searle, B. (2015c). Let's Talk Learning Analytics Framework. Retrieved September 25, 2015 from http://www.letstalklearninganalytics.edu.au/wp-content/uploads/2015/06/Lets-Talk-LearningAnalytics-Framework-Final-26-May-2015.pdf 
Let’s Talk Learning Analytics: A Framework for Implementation in Relation to Student Retention

Willcoxson, L., Manning, M., Hibbins, R., Joy, S., Thomas, J., Girardi, A., Leask, B., Sidoryn, T., Cotter, J., Kavanagh, M., Troedson, D., \& Lynch, B. (2011). The whole of university experience: Retention, attrition, learning and personal support interventions during undergraduate business studies. Surrey Hills, NSW: Australian Learning and Teaching Council. Retrieved May 10, 2015 from http://eprints.usq.edu.au/20138/

Yeado, J., Haycock, K., Johnstone, R., \& Chaplot, P. (2014). Education trust higher education practice guide: Learning from high-performing and fast-gaining institutions. The Education Trust, Washington, DC. 